\title{
A Novel Approximate Numerical Method for Solving the Evolution of the Spin Density Operators
}

\author{
Maryam Hatefi ${ }^{\mathrm{a}}$, Farrokh Sarreshtedari ${ }^{\mathrm{a}, *}$, and Mahmood Sabooni ${ }^{\mathrm{b}, \mathrm{c}}$ \\ ${ }^{\text {a }}$ Magnetic Resonance Research Laboratory, Department of Physics, College of Science, \\ University of Tehran, 143-9955961, Tehran, Iran. \\ ${ }^{b}$ Department of Physics, College of Science, University of Tehran, 143-9955961, Tehran, Iran. \\ ${ }^{\mathrm{c}}$ Institute for Quantum Computing, Department of Physics and Astronomy, Waterloo, \\ Ontario, N2L3G1, Canada.
}

*Corresponding author Email: f.sarreshtedari@ut.ac.ir

Regular paper: Received: Nov. 29, 2020, Revised: Jan. 16, 2021, Accepted: Feb. 14, 2021, Available Online: Feb. 16, 2021, DOI: 10.29252/ijop.14.2.169

\begin{abstract}
An approximate numerical method is proposed and discussed for solving the evolution of the spin density operator when the quantum system has an interaction with an external electromagnetic field. In this method by separating the relaxation and field interaction processes at small steps, instead of solving the conventional Liouville-von Neumann or Bloch differential equations, the time evolution of the density operator is efficiently obtained by a two-stage numerical algorithm. Here we have compared the results of this approach with Bloch equation results for a twolevel quantum system. The proposed approach has potential applications in calculation of the time evolution for different atomic system including nuclear or electron spin resonance systems.
\end{abstract}

KEYWORDS: Bloch equation, Liouville-von Neumann equation, Two level quantum systems, Spin density operator dynamics.

\section{I.INTRODUCTION}

The time evolution of the populations and coherencies between different atomic levels is an essential issue in atomic physics and different light-matter interaction processes [1], [2]. In this regard, density operator representation of an ensemble of atoms is a general approach in describing the atomic quantum system. Using the density operator has different advantages, which include the physical interpretation of its matrix elements that lead to the possibility of phenomenological inclusion of various physical effects into the equation of motion [1]. Some of these effects which can be considered as relaxation terms of appropriate matrix elements include decay to unobserved levels, spontaneous decay between levels, quenching collisions of active levels, incoherent pumping, etc [1]. It should be noted that the density operators for a closed system should satisfy the following requirements throughout the whole evolution [3]: (a) Hermitian, so that all the probabilities are real, (b) trace-preserving, because the sum of the probabilities over any complete set must be one, (c) positive, otherwise some probabilities might be negative. Different methods such as Liouville-von Neumann equation have been developed for the solution of the spin density operators [2-7]. In the special case of two-level systems, the time evolution of the density matrix elements can be described by the classical Bloch equation [8]. The Bloch equation was developed in 1946 to describe the motion of the expectation value of the system magnetization under the influence of control fields and longitudinal and transverse relaxations.

In this work, a novel numerical approach for the calculation of the density matrix time evolution in a multi-level system is presented 
and its results for a two-level system is compared with the conventional Bloch equation solution.

\section{TheORETICAL MODEL}

Here, we describe our computational method with notations of a two-level system in which the results can be compared with conventional Bloch solution. The density operator of a twolevel ensemble is given in equation (1).

$\hat{\rho}=\left(\begin{array}{ll}\rho_{\alpha} & \rho_{+} \\ \rho_{-} & \rho_{\beta}\end{array}\right)$

where $\rho_{\alpha}$ and $\rho_{\beta}$ stand for the population of the states, while $\rho_{+}$and $\rho_{-}$describe the coherency between them. The spin density operator can be also given by using a magnetization vector as equation (2).

$$
\begin{aligned}
\hat{\rho} & =\frac{1}{2} \hat{1}+\frac{1}{2} \frac{\hbar \gamma B^{0}}{k_{B} T} \mathbf{M} \cdot \hat{I} \\
& =\frac{1}{2} \hat{1}+\frac{1}{2} \frac{\hbar \gamma B^{0}}{k_{B} T}\left(M_{x} \hat{I}_{x}+M_{y} \hat{I}_{y}+M_{z} \hat{I}_{z}\right)
\end{aligned}
$$

where $\mathbf{M}\left(\mathbf{M}=M_{x} e_{x}^{\hat{n}}+M_{y} \hat{e}_{y}+M_{z} e_{z}^{\hat{n}}\right)$ is the system magnetization vector. The three components of the magnetization vector $M_{x}$, $M_{y}$, and $M_{z}$ are related to the four density operator matrix elements as given in equation (3) [9].

$$
\begin{aligned}
& M_{x}=4 \frac{k_{B} T}{\hbar \gamma B^{0}} \operatorname{Re}\left\{\rho_{-}\right\} \\
& M_{y}=4 \frac{k_{B} T}{\hbar \gamma B^{0}} \operatorname{Im}\left\{\rho_{-}\right\} \\
& M_{z}=2 \frac{k_{B} T}{\hbar \gamma B^{0}}\left(\rho_{\alpha}-\rho_{\beta}\right)
\end{aligned}
$$

where $B^{0}$ is the external magnetic field, $\gamma$ is the gyromagnetic ratio and $T$ is the system temperature. It should be noted that the longitudinal component $M_{z}$ is proportional to the population difference between the two states and the transverse components $M_{x}$ and $M_{y}$ are related to the coherences between the states [9]. For such a two-level system, the
Bloch equation which gives a general framework for treating the simultaneous effects of relaxation, electromagnetic fields, and resonance offset, describe the components of the magnetization vector as equation (4) [9].

$$
\begin{aligned}
& \frac{d}{d t}\left(\begin{array}{l}
M_{x} \\
M_{y} \\
M_{z}
\end{array}\right)= \\
& \left(\begin{array}{ccc}
0 & -\Omega^{0} & \omega_{\text {nut }} \sin \phi \\
\Omega^{0} & 0 & -\omega_{\text {nut }} \cos \phi \\
-\omega_{\text {nut }} \sin \phi & \omega_{\text {nut }} \cos \phi & 0
\end{array}\right)\left(\begin{array}{l}
M_{x} \\
M_{y} \\
M_{z}
\end{array}\right)-\left(\begin{array}{c}
T^{-1}{ }_{2} M_{x} \\
T^{-1}{ }_{2} M_{y} \\
T_{1}^{-1}\left(M_{z}-1\right)
\end{array}\right)
\end{aligned}
$$

where $\Omega^{0}$ is the frequency detuning, $\omega_{\text {nut }}$ is the nutation frequency of the electromagnetic (RF) field, $\phi$ is the phase of the RF field, and $T_{1}$, and $T_{2}$ are the spin-lattice and spin-spin relaxation times, respectively. Our method for the calculation of the density operator evolution is based on the fact that in the presence of the electromagnetic field when the relaxation is ignored, the density operator can be found by applying the appropriate field propagator. This is while, when there is no electromagnetic field, the matrix elements of the density operator would be relaxed to their equilibrium values. In this regard, we divide the interaction time to small steps and, in each step, we separately apply the two mentioned evolution mechanisms. For example, if we assume the time steps as $t_{1}, t_{2}, t_{3}, \ldots, t_{n}$, during each $t_{i}$, we apply the electromagnetic wave to the ensemble (rotation period) and then during the same time we let the system to be relaxed (relaxation period). This sequence continues to the end of the interaction time. During the rotation period when we apply the electromagnetic field, the Hamiltonian of the interaction in the rotating frame may be written as equation (5).

$$
\hat{H}=\Omega^{0} \hat{I}_{z}+\omega_{\text {nut }}\left(\hat{I}_{x} \cos \left(\varphi_{p}\right)+\hat{I}_{y} \sin \left(\varphi_{p}\right)\right)
$$

It can be shown that this Hamiltonian can be written as equation (6).

$$
\hat{H}=\omega_{e f f} \hat{R}_{z}\left(\varphi_{p}\right) \hat{R}_{y}\left(\theta_{p}\right) \hat{I}_{z} \hat{R}_{y}\left(-\theta_{p}\right) \hat{R}_{z}\left(-\varphi_{p}\right)
$$


Where $\hat{R}_{j}$ is the propagator of the operator $\hat{I}_{j}$ which is defined as $\hat{R}_{j}\left(\beta_{p}\right)=\exp \left\{-i \beta_{p} \hat{I}_{j}\right\}$. The effective rotation frequency is $\omega_{\text {eff }}=\left\{\left(\omega_{\text {nut }}\right)^{2}+\left(\Omega^{0}\right)^{2}\right\}^{\frac{1}{2}}$, and $\theta_{p}$ is the tilt of the rotation axis away from the $\mathrm{z}$-axis and is given by $\theta_{p}=\arctan \left(\omega_{\text {nut }} / \Omega^{0}\right)$. The corresponding propagator for the Hamiltonian of the equation (6) can be given as equation (7).

$$
\hat{R}_{t o t}\left(\beta_{p}\right)=\hat{R}_{z}\left(\varphi_{p}\right) \hat{R}_{y}\left(\theta_{p}\right) \hat{R}_{z}\left(\beta_{p}\right) \hat{R}_{y}\left(-\theta_{p}\right) \hat{R}_{z}\left(-\varphi_{p}\right)
$$

where $\beta_{p}$ is the flip angle and equals to $\beta_{p}=\omega_{\text {eff }} t$. Using this propagator which is written as a product of five rotations about orthogonal axes, the evolution of the density operator can be obtained by sandwiching of the density operators between $\hat{R}_{t o t}\left(\beta_{p}\right)$ and $\hat{R}_{t o t}\left(-\beta_{p}\right)$. During the relaxation period, we assume that the quantum system relaxes to its thermal equilibrium. Based on quantum statistical mechanics, in thermal equilibrium at temperature $T$, the coherences between the states are all zero and the populations obey the Boltzmann distribution. For an isolated spin$1 / 2$ ensemble the thermal equilibrium density operator is therefore given by equation (8).

$$
\rho^{e q}=\left(\begin{array}{cc}
\frac{1}{2}+\frac{1}{4} \frac{\hbar \gamma B^{0}}{k_{B} T} & 0 \\
0 & \frac{1}{2}-\frac{1}{4} \frac{\hbar \gamma B^{0}}{k_{B} T}
\end{array}\right)
$$

In the relaxation period, the density matrix elements change as equations (9).

$$
\begin{aligned}
& \rho_{\alpha}(t)=\left(\rho_{\alpha}^{0}-\rho_{\alpha}^{e q}\right) e^{-t / T_{1}}+\rho_{\alpha}^{e q} \\
& \rho_{\beta}(t)=\left(\rho_{\beta}^{0}-\rho_{\beta}^{e q}\right) e^{-t / T_{1}}+\rho_{\beta}^{e q} \\
& \rho_{-}(t)=\rho_{-}^{0} \exp \left\{\left(i \Omega^{0}-\frac{1}{T_{2}}\right) \tau\right\} \\
& \rho_{+}(t)=\rho_{+}^{0} \exp \left\{\left(-i \Omega^{0}-\frac{1}{T_{2}}\right) \tau\right\}
\end{aligned}
$$

where $T_{1}$ and $T_{2}$ are the longitudinal and transverse relaxation time constants. It should be noted that for simplicity we have considered $T_{2}=2 T$ and $\gamma=T_{2}^{-1}$ in our calculations.

\section{III.RESULT AND DISCISSION}

We have compared the calculation of the magnetization evolution using the conventional Bloch equations and the proposed approach for different initial magnetization and system parameters including frequency detuning, nutation frequency and relaxation rate.

Figure 1 shows the comparison results for the time evolution of different magnetization components with a typical group of parameters in a time interval with a flip angle of $10 \pi$. In this calculations, the initial magnetization is $\left[M_{x 0}, M_{y 0}, M_{z 0}\right]=[1,0,1]$, the relaxation rate is $\gamma=1 \times 10^{5}\left(s^{-1}\right)$, the frequency detuning is $\Omega^{0}=1 \times 10^{3}(\mathrm{~Hz})$ and the nutation frequency is considered as $\omega_{n u t}=1 \times 10^{6}(\mathrm{~Hz})$. Figures 1(a), (b) show the Bloch and the proposed approach with 500 steps solutions, respectively. For better comparison, fig. 1(c) simultaneously show the results of the two methods, while the number of steps is decreased to 200 to emphasis the differences. It should be noted that as expected, the $M_{z}$ and $M_{y}$ has exponentially decreasing oscillations while the $M_{x}$ which is the magnetization component parallel to the direction of the applied field has a decaying behavior. Figure 2 shows the comparison of the Bloch solution and the proposed approach for obtaining the $M_{z}$ component of the magnetization at various relaxation rates. In these calculations, the initial magnetization is considered as $\left[M_{x 0}\right.$, $\left.M_{y 0}, \quad M_{z 0}\right]=[0, \quad 0,1]$, the detuning is $\Omega^{0}=1 \times 10^{3}(\mathrm{~Hz})$, the nutation frequency is $\omega_{\text {nut }}=1 \times 10^{6}(\mathrm{~Hz})$, and the number of steps is equal to 200 . 

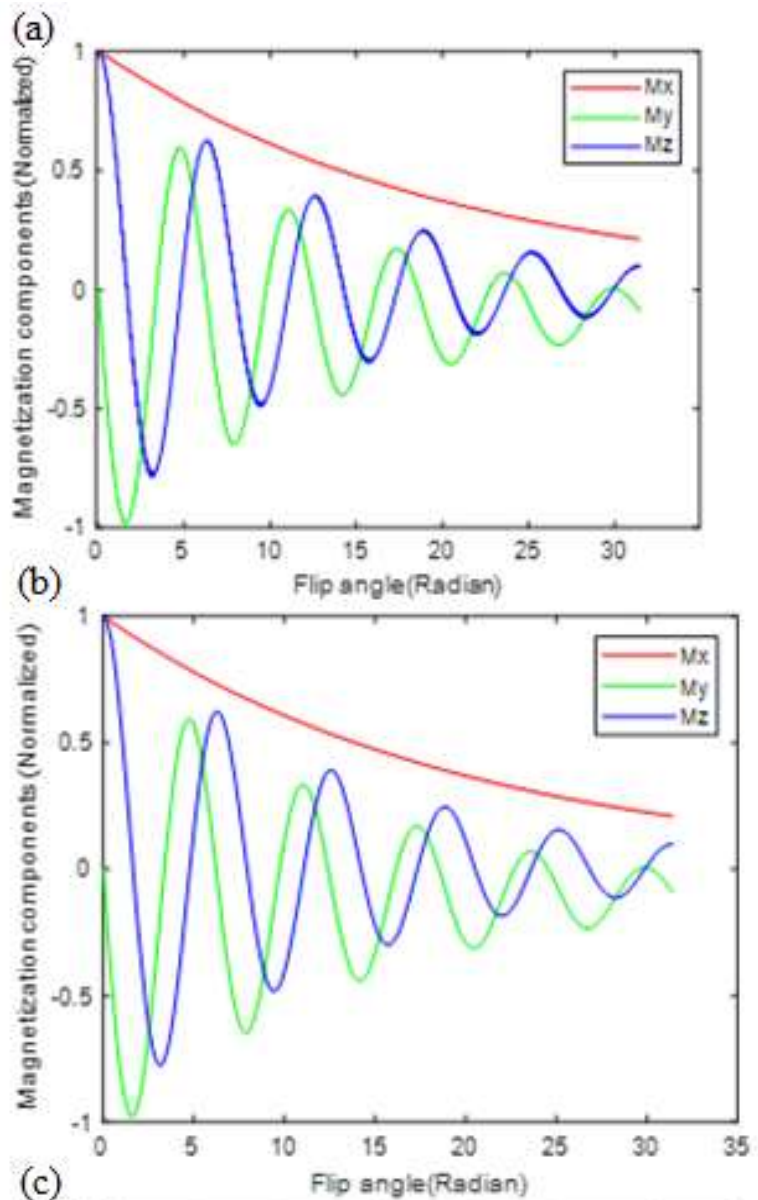

(c)

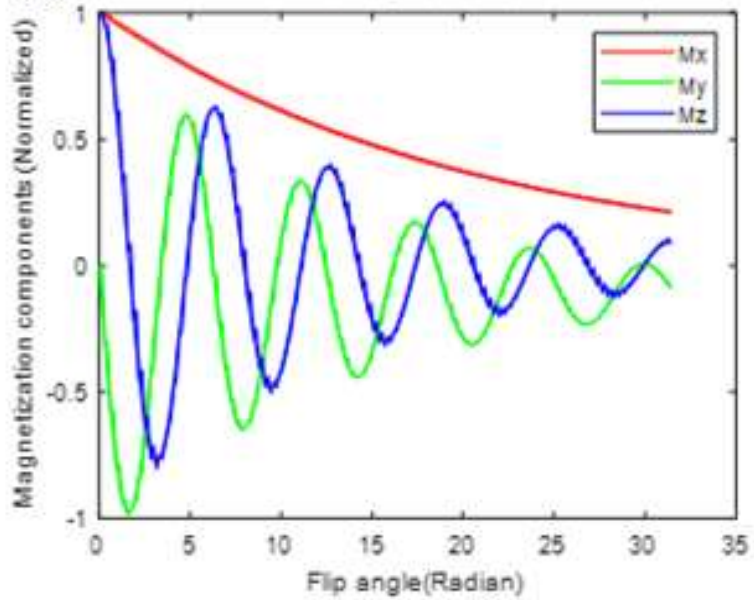

Fig. 1. The evolution of different magnetization components. The initial magnetization is $\left[M_{x 0}, M_{y 0}\right.$, $\left.M_{z 0}\right]=\left[\begin{array}{lll}1, & 0, & 1\end{array}\right], \quad \gamma=1 \times 10^{5}\left(s^{-1}\right), \quad \Omega^{0}=1 \times 10^{3}(\mathrm{~Hz})$, $\omega_{\text {nut }}=1 \times 10^{6}(\mathrm{~Hz})$, calculated by (a) the proposed approach with 500 steps, (b) the Bloch solution, (c) the both results (with 200 steps).

It is clear that both approaches have the same results. This is while in our approach, because of the low number of steps, there are added fluctuations. The effect of the number of steps on the calculation results is investigated in fig. 3. This figure shows the calculation of the magnetization evolution using the proposed approach with different step numbers. In these calculations, the considered system parameters are the same values used in the previous figure with $\gamma=1 \times 10^{5}\left(s^{-1}\right)$.
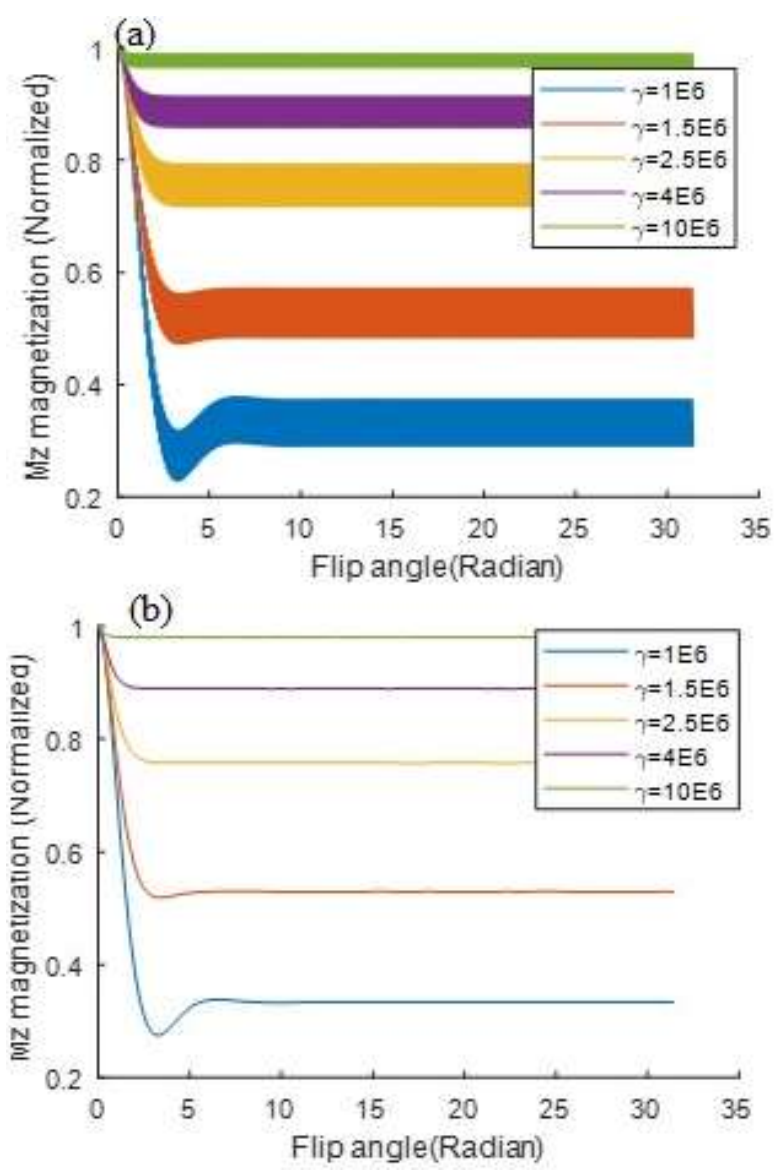

Fig. 2. The calculation results of the magnetization evolution. When the initial magnetization is $\left[M_{x 0}\right.$, $\left.M_{y 0}, \quad M_{z 0}\right]=[0, \quad 0, \quad 1], \quad \Omega^{0}=1 \times 10^{3}(\mathrm{~Hz})$, $\omega_{n u t}=1 \times 10^{6}(\mathrm{~Hz})$, calculated by (a) the proposed approach, (b) the Bloch solution.

It is obvious that by increasing the step numbers, the results of the proposed approach become more similar to the result of the Bloch equations. This is because increasing the step numbers is equivalent to decreasing the periods of free rotation and free relaxation which make the calculation more accurate. It should be noted that in the proposed method, the order of the two parts of the calculation has a small effect on the results. 

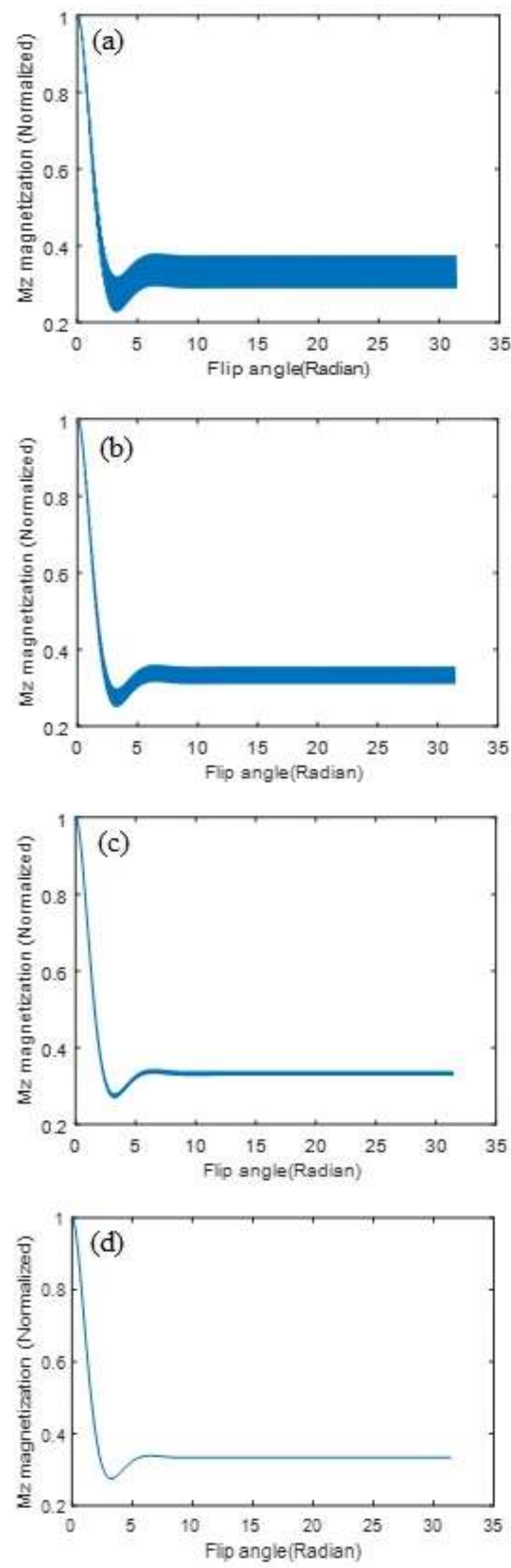

Fig. 3. The calculation results of the magnetization evolution when the initial magnetization is $\left[M_{x 0}\right.$, $\left.M_{y 0}, M_{z 0}\right]=[0,0,1], \gamma=1 \times 10^{6}\left(s^{-1}\right), \Omega^{0}=1 \times 10^{3}(H z)$, $\omega_{\text {nut }}=1 \times 10^{6}(\mathrm{~Hz})$, with different step numbers (a) 500 steps, (b) 1000 steps, (c) 5000 steps, (d) The conventional Bloch solution

Figure 4 shows the solution of the same problem with a different order for applying of the relaxation and rotation processes. In this figure, the calculation is done with small number of steps (100 steps) for a clear presentation of the difference. It is evident that solving the problem with both orders and averaging on their result obviously improve the calculation accuracy.

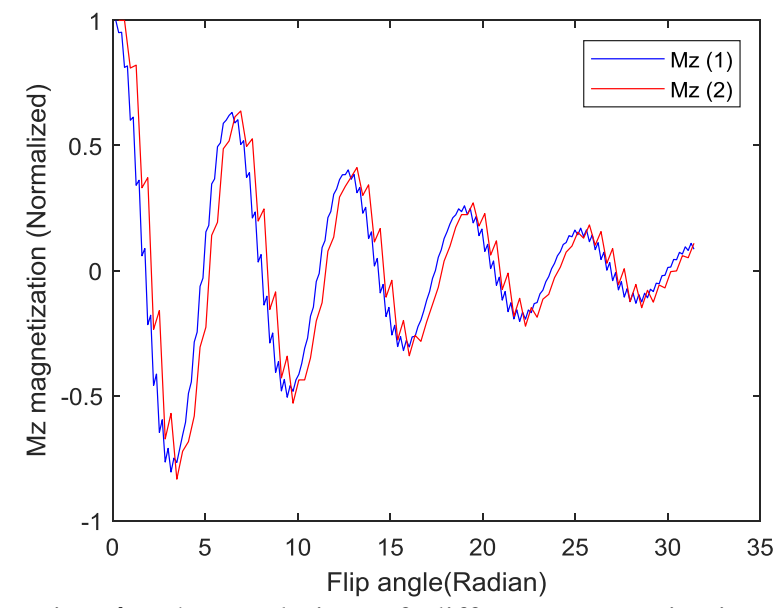

Fig. 4. The evolution of different magnetization components. When the initial magnetization is $\left[M_{x 0}\right.$, $\left.M_{y 0}, M_{z 0}\right]=[1,0,1], \gamma=1 \times 10^{5}\left(s^{-1}\right), \Omega^{0}=1 \times 10^{3}(H z)$, $\omega_{\text {nut }}=1 \times 10^{6}(\mathrm{~Hz})$, step numbers $=100$. The blue line: first rotation and then relaxation, The red line: first rotation and then relaxation.

The results of the proposed algorithm for the spin dynamics solution is matched with the Bloch solution for a wide range of system parameters. For investigation of the matching characteristic between the two approaches, we have changed the system parameters and obtained the difference between the two solutions. Figure 5 shows the error percentage between the results of the steady state value of the proposed method and Bloch solution for different relaxation rates and nutation frequencies. Different parts of this figure (a-d), show the error result for different frequency detuning. 
Although the error percentage is small for a wide range of parameters, it is evident that by increasing the detuning, the error also increases. This is while for different detuning, the error is a maximum at low relaxation rates.
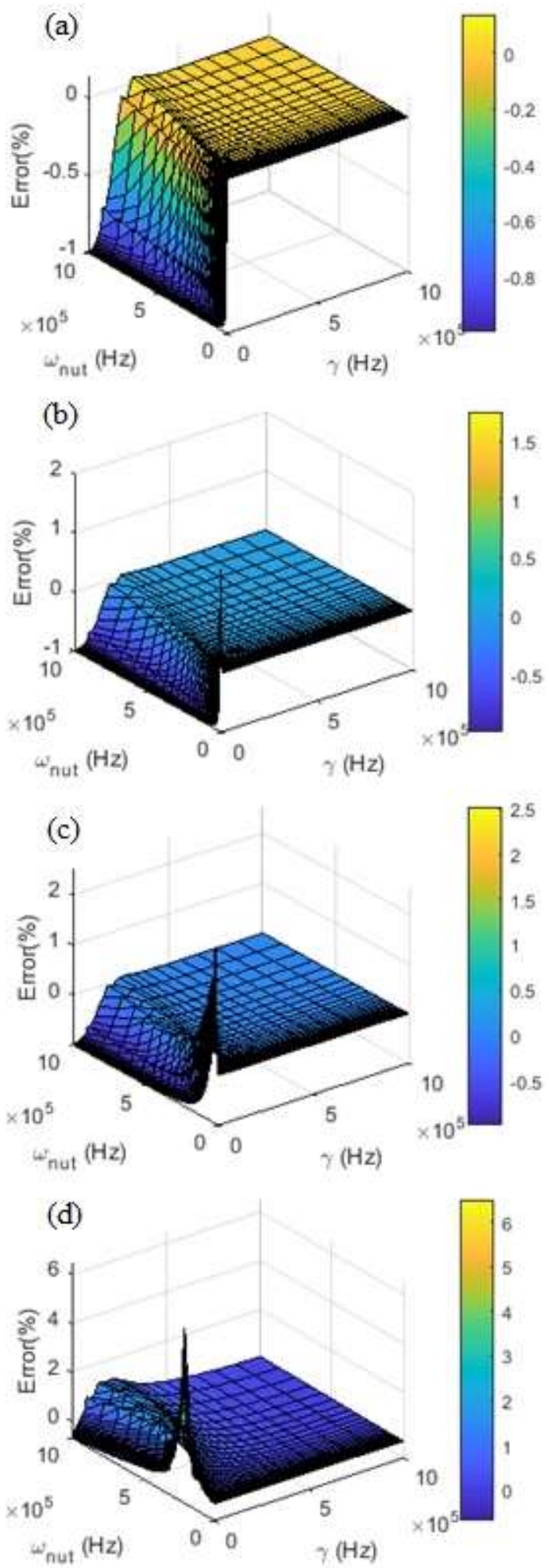

Fig. 5. The calculated error between the results of proposed approach and the Bloch solution for the

$$
\begin{aligned}
& \text { initial magnetization of }\left[M_{x 0}, M_{y 0}, M_{z 0}\right]=[0,0,1] \text {, } \\
& \text { and at different } \omega_{\text {nut }} \text { and } \gamma \text {. The considered } \\
& \begin{array}{lll}
\text { detuning is } \quad \text { (a) } \Omega^{0}=1 \times 10^{2}(H z), & \text { (b) } \\
\Omega^{0}=1 \times 10^{3}(\mathrm{~Hz}), & \text { (c) } \Omega^{0}=1 \times 10^{4}(\mathrm{~Hz}), & \text { (d) } \\
\Omega^{0}=1 \times 10^{5}(\mathrm{~Hz}) . & &
\end{array}
\end{aligned}
$$

The reason for this behavior is that at low relaxation rates, the steady state solution would be obtained in large flip angles (times). This is while we have considered a finite time interval for acquiring the steady state values. It should be also noted that the solution time of this method is remarkably short even for very small steps. This is because the calculation process includes matrix multiplication and obtaining of the exponentially changing variables. We believe that this method could be useful for efficient and approximate solutions of different complex atomic systems.

\section{IV.CONCLUSION}

Here, we have presented an approximate method to calculating spin dynamic evolutions. In this method, the time evolution of the spin density operator is calculated by dividing the total time interval into very small steps. In each step, two successive processes are considered. First ignoring the relaxation, the time evolution of the density operator in the presence of external field is obtained and then ignoring the effect of external field, the relaxation of the populations and the coherences of the density operator is calculated. For a two-level system, we have compared the results of this approach with the solution of the classical Bloch equation. It is shown that considering small enough steps in our approach, a very good agreement can be achieved between these two methods. The presented approach is obviously not limited to the two-level systems but can be used for multi-level and more complex systems.

\section{REFERENCES}

[1] S. Stenholm, Foundations of Laser Spectroscopy, Mineola, New York, 2005.

[2] M. Auzinsh, D. Budker, and S. Rochester, Optically Polarized Atoms: Understanding 
light-atom interactions, Oxford University press, 2010.

[3] R.S. Whitney, "Staying positive: going beyond lindblad with perturbative master equations," J. Phys. A, Vol. 41, pp. 175304 (1-18), 2008.

[4] H.P. Breuer, F. Petruccione, The theory of open quantum systems, Oxford University press, 2002.

[5] G. Lindblad, "On the generators of quantum dynamical semigroups," Commun. Math. Phys. Vol. 48, pp. 119-130, 1976.

[6] H. Tian and G.H. Chen, "An efficient solution of Liouville-von Neumann equation that is applicable to zero and finite temperatures," J. Chem. Phys, Vol. 137, pp. 204114, (1-6), 2012.

[7] G. Mazzi, "Numerical Treatment of the Liouville-von Neumann Equation for Quantum Spin Dynamics," Ph.D thesis, University of Edinburgh, 2010.

[8] F. Bloch, "Nuclear Induction," Phys. Rev, Vol. 70, pp. 460 (1-16), 1946.

[9] I.I. Mazin and M.D. Johannes, Spin Dynamics, John Wiley \& Sons Ltd, $2^{\text {nd }}$ Ed, 2008.

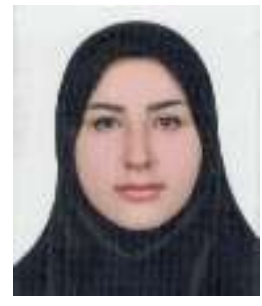

Maryam Hatefi was born in Iran in 1994. She received the B.Sc. degrees in Physics from Isfahan University, Isfahan, Iran, in 2016 and M.Sc. degree in Atomic Physics from Tehran University, Tehran, Iran, in 2020. Her research interest includes Optics, lasers and amplifiers.

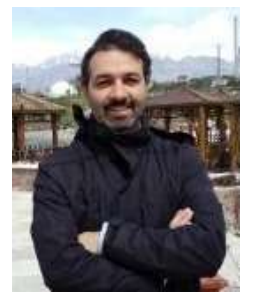

Farrokh Sarreshtedari was born in Iran in 1982. He received the B.Sc., M.Sc. and Ph.D. degrees from Sharif University of Technology, Tehran, Iran, in 2004, 2006 and 2012, respectively, all in electrical engineering. He is currently a faculty member in Department of Physics, University of Tehran. He has set up the "Magnetic Resonance Research Laboratory (MRRL)" at University of Tehran in 2015, which he directs since then. His research interests include Magnetic resonance physics, lasers and light-matter interactions.

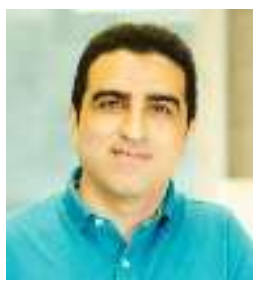

Mahmood Sabooni received his $\mathrm{PhD}$ degree from Quantum Information Group, Atomic Physics Department at Lund University of Technology, Lund, Sweden in April 2013 while his MSc. was awarded in Solid State Physics from Shahrood University of Technology in June 2005. From 2005 to 2008 he was faculty member in Azad University (Shahrood branch). He has appointment as a senior researcher at institute for quantum computing (IQC), Waterloo, Canada since March 2015 after a postdoc at Max Planck institute for Quantum Optics (MPQ), Garching, Munich, Germany starts at September 2013. His main interest is mostly on experimental light-matter interaction in the quantum regime. During his $\mathrm{PhD}$, his main focus was investigating ways to map the quantum state of light quanta (photon) to an ensemble of atom and retrieve it back as efficient as possible. By starting his postdocs in Munich (MPQ) and Waterloo (IQC), he extends his knowledge about quantum information processing to a new physical implementation (cavity-quantum electrodynamics). 
THIS PAGE IS INTENTIONALLY LEFT BLANK. 